\title{
Étude ethnobotanique et phytochimique de Momordica charantia Linn (Cucurbitaceae) à Cotonou au Bénin
}

\author{
Roch Christian Johnson ${ }^{1}$, Eustache Enock Houéto ${ }^{1 *}$, Gratien Boni ${ }^{1}$, Wilfrid Hinnoutondji Kpètèhoto ${ }^{1}$,' \\ Victorien Dougnon ${ }^{2}$, Elias Pognon ${ }^{3}$, Fidèle Assogba ${ }^{4}$, Frédéric Loko ${ }^{2}$, Michel Boko ${ }^{1}$, Joachim Gbénou ${ }^{4}$ \\ ${ }^{1}$ Laboratoire d'Hygiène, d'Assainissement, de Toxicologie et de Santé Environnementale (HECOTES), du Centre \\ Interfacultaire de Formation et de Recherche en Environnement pour le Développement Durable (CIFRED) de l'Université \\ d'Abomey-Calavi (UAC / Bénin), 01, PO Box 1463, Cotonou, Benin. \\ ${ }^{2}$ Laboratoire de Recherche en Biologie Appliquée (LARBA) à l'École Polytechnique d'Abomey-Calavi (EPAC) de \\ l'Université d'Abomey-Calavi (UAC / Bénin). \\ ${ }^{3}$ Laboratoire de Contrôle de Qualité des Eaux et Aliments (LCQEA) du Ministère de la Santé Publique (MSP / Bénin). \\ ${ }^{4}$ Laboratoire de Pharmacognosie et des Huiles Essentielles (LAPHE) de l'Institut des Sciences Biomédicales Appliquées \\ (ISBA) de l'Université d'Abomey-Calavi (UAC / Bénin). \\ Auteur correspondant : rochjohnson@yahoo.fr.Téléphones : (00229) 96221132
}

Original submitted in on 22nd July 2016. Published online at www.m.elewa.org on $31^{\text {st }}$ October 2016 http://dx.doi.org/10.4314/jab.v106i1.4.

\section{RÉSUMÉ}

Objectif : La présente étude, sur Momordica charantia Linn (Cucurbitaceae), communément appelé margose une plante très utilisée pour le traitement de nombreuses maladies, est menée dans la ville de Cotonou au Bénin. Elle vise à vérifier la portée scientifique des usages empiriques que fait la population de cette plante, afin de promouvoir son utilisation sans risque.

Méthodologie et résultats : Une étude ethnobotanique est réalisée auprès de 32 vendeurs des trois marchés de plantes médicinales les plus fréquentés de Cotonou. Elle est complétée par une. exploration phytochimique, associée au dosage des métaux lourds et de quelques éléments nutritifs. Les vertus connues de la plante, recensées lors de l'étude ethnobotanique, sont corrélées avec les fractions actives mises en évidence par l'étude phytochimique qui révèle la présence des tanins, flavonoïdes, dérivés anthracéniques, alcaloïdes, stéroïdes, et mucilages. Le plomb et le cadmium sont aussi détectés à des concentrations d'environ neuf et six fois, respectivement, supérieures aux normes de l'Organisation Mondiale de la Santé Conclusion et application des résultats : Cette étude a permis de connaitre les usages empiriques que fait la population de cette plante puis de les lier aux substances actives présentes. Ces résultats constituent un outil indispensable dans la mise en place d'une base de données pour la validation scientifique des connaissances traditionnelles des plantes au Bénin. Toutefois, ces recherches révèlent les risques de contamination au plomb et cadmium auxquels la population est soumise dans l'utilisation de Momordica charantia.

Mots clés : Momordica charantia ; Ethnobotanique ; Ecotoxicologique ; Cotonou ; Benin. 
Ethnobotanical and phytochemical study of Momordica charantia Linn (Cucurbitaceae) in Cotonou Benin

\begin{abstract}
Objective: This study, on Momordica charantia Linn (Cucurbitaceae), commonly known as margose a plant widely used for the treatment of many diseases, is conducted in. Cotonou the capital city of Benin. It aims to verify the scientific scope of the empirical uses of this plant.and to promote its safe use

Methodology and Results: An ethnobotanical study was conducted among 32 vendors in the three main markets of medicinal plants in Cotonou. In addition, a phytochemical exploration, associated with the determination of heavy metals and some nutrients were also been conducted on this plant. During the ethnobotanical study the known virtues of the plant, are correlated with the active fractions revealed by the phytochemical screening (tannins, flavonoids, anthracene derivatives, alkaloids, steroids, and mucilage). Lead and Cadmium were also detected with concentrations of about nine and six times, respectively, higher than the World Health Organization standards for human nutrition.

Conclusion and application of results: This study revealed the correlation between the empirical uses of Mormodica Charantia and the phytochemical study. This study results highlight the importance to establish a database for validation of traditional knowledge of population related to the plants uses However, this research shows the risk of contamination with lead and cadmium which the population is subjected in the use of. Momordica charantia.
\end{abstract}

Keywords : Momordica charantia ; Ethnobotany ; Ecotoxicological ; Cotonou ; Benin.

\section{INTRODUCTION}

Au travers des âges, l'homme a pu compter sur la nature pour subvenir à ses besoins de base.(Lhuillier, 2007). L'utilisation des plantes à des fins médicinales, remonte à la préhistoire et cette tradition a été transmise de génération en génération (Soh et al., 2007). En Afrique, l'utilisation de la médecine traditionnelle, varie de $70 \%$ au Bénin à 90 $\%$ au Burundi et en Éthiopie (OMS, 2013). Dans l'optique de la valorisation de la médecine traditionnelle, les plantes médicinales qui présentent des utilisations thérapeutiques intéressantes sont identifiées et étudiées pour aider les populations démunies à tirer un réel avantage de l'usage des plantes de leur pharmacopée (Kougnimon et al., 2015). Par ailleurs, les effets secondaires des molécules de synthèse ont conduit ces dernières années à l'utilisation des plantes médicinales à des fins thérapeutiques (Kpodékon et al., 2014). C'est dans le but de promouvoir l'utilisation sans risque de Momordica charantia, margose (figure 1) au sein de la population, que la présente étude intitulée. a été initiée.

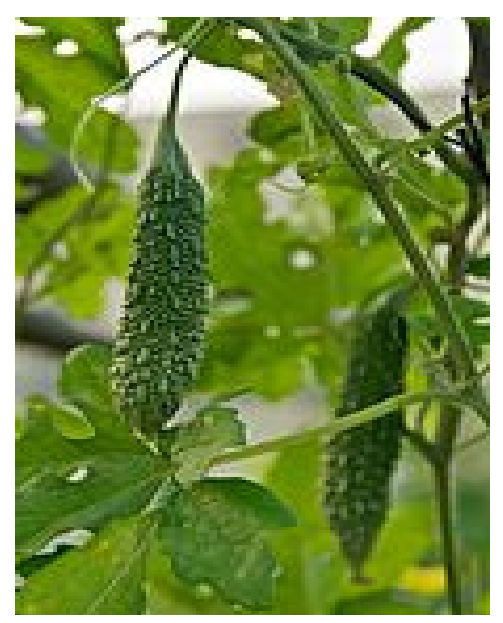

Figure 1: Plante de Momordica charantia source https://fr.wikipedia.org/wiki/Momordica_charantia 


\section{METHODOLOGIE}

Cotonou, la plus grande ville du Bénin a servi de cadre pour cette étude. Située entre $6^{\circ} 21^{\prime} 36^{\prime \prime}$ de latitude Nord et $2^{\circ} 26^{\prime} 24^{\prime \prime}$ longitude Est sur le cordon littoral, comprise entre le lac Nokoué et l'Océan Atlantique, et seule commune du département du Littoral. Le climat est de type subéquatorial avec l'alternance de deux saisons pluvieuses et deux saisons sèches. La pluviosité moyenne est de $1200 \mathrm{~mm}$ par an, avec une température moyenne annuelle variant de $26^{\circ} \mathrm{C}$ à $28^{\circ} \mathrm{C}$ et une humidité relative supérieure et une humidité relative supérieure à $60 \%$ (Akomagni, 2006). Ce travail a consisté à réaliser deux types d'étude: un relevé d'enquête ethnobotanique et un criblage phytochimique associé au dosage de quelques éléments nutritifs, du plomb et du cadmium. Au cours de nos investigations sur le terrain, un questionnaire a été administré à 32 vendeurs des trois marchés de plantes médicinales les plus fréquentés de Cotonou. La fiche questionnaire comporte des questions précises sur l'informateur (âge, sexe, région d'origine.) et la plante objet de l'étude (nom vernaculaires, type de maladie traitée, partie utilisée, mode de préparation etc.). Un recensement des vendeurs a été fait au cours d'une phase de pré enquête et proportionnellement au nombre d'enquêtés désirés, un quota a été retenu par marché. Les vendeurs sont choisis en tenant compte des critères ci-après :

Le premier vendeur, à l'entrée principale du marché est choisi, s'il obéit aux critères ci-après, sinon on passe au suivant avant d'effectuer un bond de 4 pour le

\section{RÉSULTATS ET DISCUSSION}

De l'étude ethnobotanique: Les résultats de cette étude ethnobotanique sont axés d'une part sur les divers usages de Momordica charantia, les plantes médicinales usuelles, les sources d'approvisionnement et d'autre part sur les organes végétaux utilisés, les modes de préparation, les voies d'administration. Les résultats des enquêtes dans les marchés se présentent comme suit :

- $\quad$ Plantes médicinales usuelles à Cotonou : Dans le but de comparer la fréquence d'utilisation de Momordica charantia à celle de quatre autres plantes médicinales très utilisées à Cotonou, Momordica charantia $(16,66 \%)$ vient en quatrième position après Senna rotondifilia $(32,07 \%)$.. Carica papaya $(21,31 \%)$ et marché Dantokpa et Vêdoko, et un bond de 3 pour le marché de Vossa ;

La richesse de l'étalage du vendeur de plantes médicinales est prioritaire ;

- $\quad$ La fraicheur des espèces vendues en général et de Momordica charantia en particulier.

Les informations recueillies sont inscrites dans une base de donnés puis traitées et analysées statistiquement à l'aide du logiciel de Microsoft Office "Excel ". Concernant l'étude phytochimique, les substances à rechercher au laboratoire sont les métabolites secondaires de la plante. Ces tests sont réalisés sur la base des réactions (coloration et précipitation) différentielles des principaux groupes de composés chimiques contenus dans la plante selon la méthode de Houghton et Rama (1998) revue et adaptée aux conditions de laboratoire de pharmacognosie des Huiles essentielles de l'Institut des sciences Biomédicales appliquées. de l'Université d'Abomey Calavi (Benin), rapporté par Dougnon.et al. (2012). Le dosage des Éléments Traces Métalliques (ETM) est fait au spectrophotomètre d'absorption moléculaire DR 6000. Le protocole utilisé est celui défini par Rothery et al., (1988) adapté aux conditions du laboratoire d'Hygiène, d'Assainissement, de Toxicologie et de Santé Environnementale (HECOTES). Pour la validité des résultats, les analyses ont été reprises au laboratoire de Contrôle de Qualité des Eaux et aliments (LCQEA).

Moringa lucida benth (19,23\%). Ce résultat s'oppose à celui de Okafor et al., (1999). En effet, au terme de leurs travaux, en considérant le nombre de maladies traités par chaque plante, Carica papaya (12) vient en tête suivi de Ocimum gratissimum (11), puis du Citrus aurantifolia et du Psidium guajava (7 chacun). Cette variation dans les résultats est due au fait que deux des trois (03) plantes devançant Momordica charantia sont des plantes antipaludiques connues (Montcho, 2012). La divergence des zones d'étude pourrait expliquer également cette variation observée dans les résultats. Quant à Ocimum gratissimum, il est surtout utilisé comme légume. 


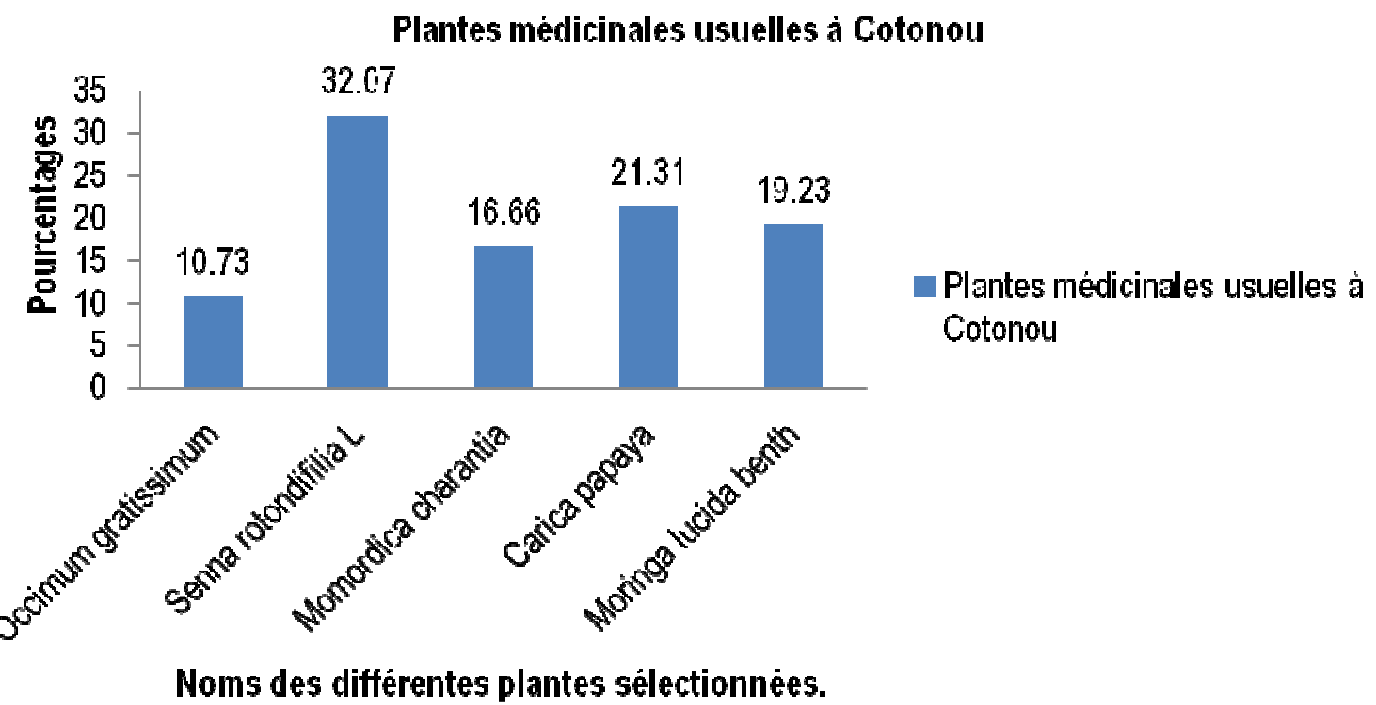

Figure 2 : Pourcentage de vente des plantes médicinales usuelles à Cotonou

- $\quad$ Source d'approvisionnement des plantes: L'analyse de cette figure 2 montre que la vallée de l'Ouémé (39\%), est la principale source d'approvisionnement de Momordica Charantia suivi de Zè $(26 \%)$, d'Allada (18\%), de Ouidah (6\%), de Godomey (7 $\%$ ) et d'Adjarra (4\%). Ceci pourrait s'expliquer par le fait que la vallée de l'Ouémé regorge encore d'une biodiversité remarquable. De plus la faible urbanisation des communes la composant, favorise le développement de la flore sauvage. La vallée de l'Ouémé est également un espace assez grand car regroupant quatre communes. Quant à Zè et Allada leur proximité de Cotonou fait d'eux des sources d'approvisionnement prisées. La présence de cette plante sur ces espaces serait aussi due à la qualité des sols. Le marché de Godomey constitue un marché de transit (Montcho, 2012). La position de la commune d'Adjarra serait due à la forte urbanisation actuelle dans cet espace.

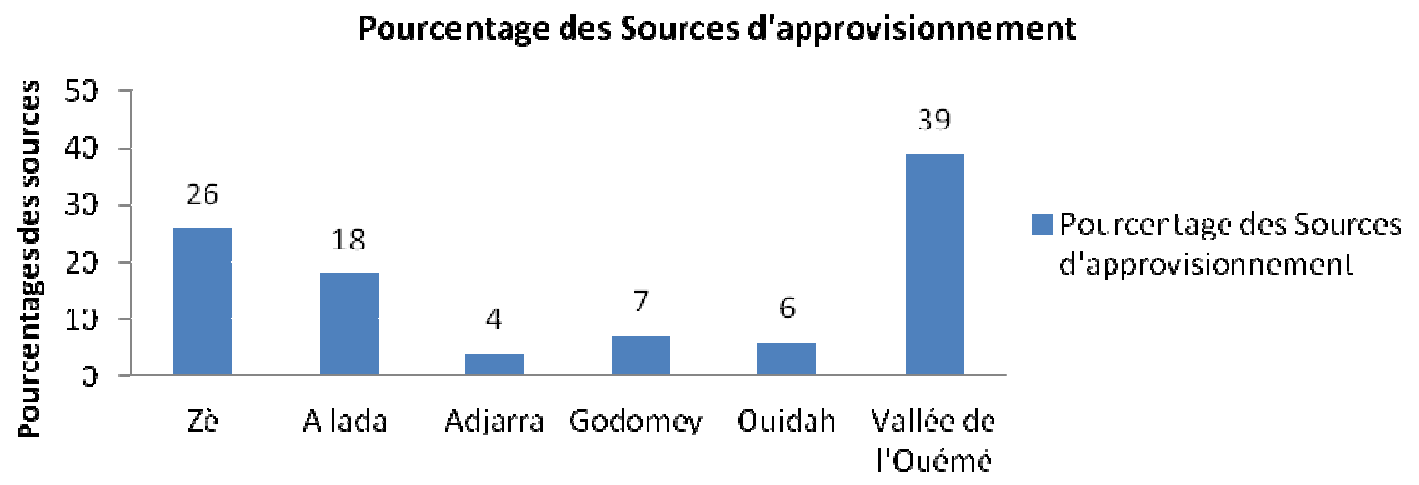

Sites d'approvisionnement

Figure 3 : Sources d'approvisionnement de Momordica charantia

- Utilisation des différents organes de la plante pour les soins des maladies : De l'examen de la figure 3 , il ressort qu'au total, neuf (9) parties de plantes sont utilisées en médecine traditionnelle notamment les racines, la tige, les feuilles, les fruits, les graines, la partie aérienne, la plante entière, la fleur et la pousse. A Cotonou, la plante entière est plus usitée $(60 \%)$ que les fruits $(20 \%)$, les feuilles et la tige $10 \%$. Ces résultats s'opposent à ceux de Tahri et al., (2012), qui dans ses études sur les plantes médicinales au Maroc, constate 
que, les feuilles sont plus utilisées, suivies des fruits. Même remarque faite par Bitsindou, (1986) qui argumente en disant que la feuille est le siège de la photosynthèse et parfois du stockage de certains métabolites responsables des propriétés biologiques. De même, Salhi et al., (2010), affirme que la proportion d'utilisation des feuilles est souvent élevée à cause de l'aisance de la récolte. Or, l'espèce dont nous disposons, présente de petites feuilles, difficiles à récolter. Par ailleurs, dans nos enquêtes, il n'a été fait cas, nulle part, que la plante sert de légume à Cotonou. C'est seulement Agbankpé et al., (2014) qui a parlé de Momordica charantia, en tant que légume feuille qui est une liane prélevée à l'état sauvage. Ceci n'est pas un facteur motivant pour amener à la culture de l'espèce.

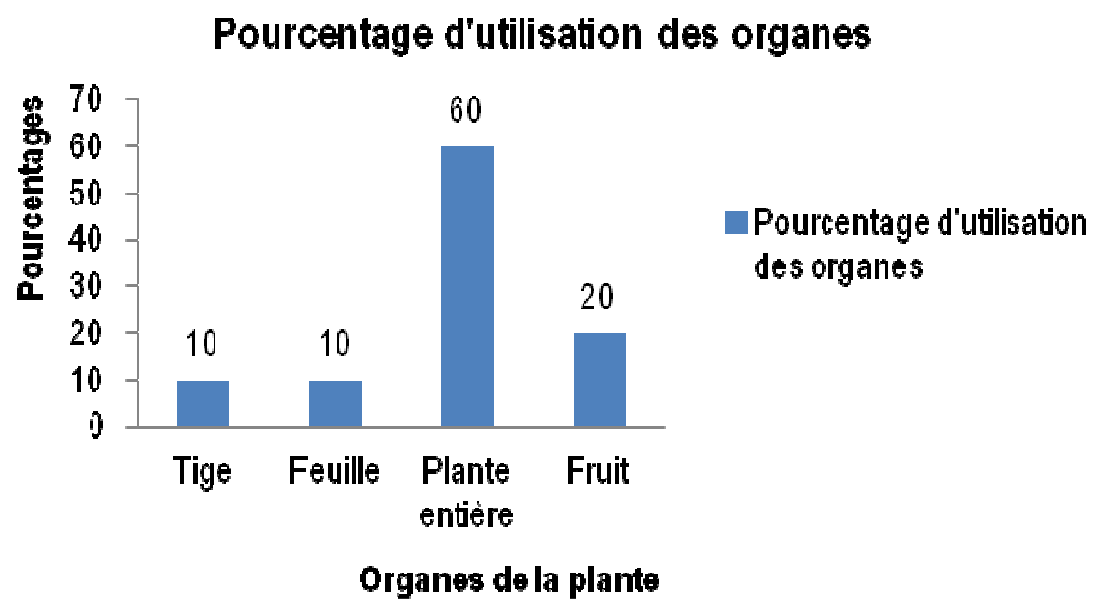

Figure 4 : Différents organes utilisés de la plante par niveau d'importance à Cotonou

- $\quad$ Mode de préparation : Cette figure montre qu'à Cotonou, la décoction et la macération sont les deux modes de préparation connues, d'après nos enquêtes, avec des taux respectivement de $60 \%$ et $40 \%$. Ceci est confirmé par Salhi et al; (2010) qui affirme que «la décoction aqueuse $(37,6 \%)$, l'infusion $(24,7 \%)$ et la poudre $(25,9 \%)$ sont les modes de préparation les plus utilisés.
Ceci pourrait s'expliquer par le fait que ce soit des méthodes plus faciles d'utilisation ou l'insuffisance de connaissance plus approfondies, des recettes de cette plante. Ce pourcentage montre que la population locale préfère la décoction et la trouve adéquate pour réchauffer le corps et désinfecter la plante (Lahsissène et al., 2010).

\section{Mode de préparation}

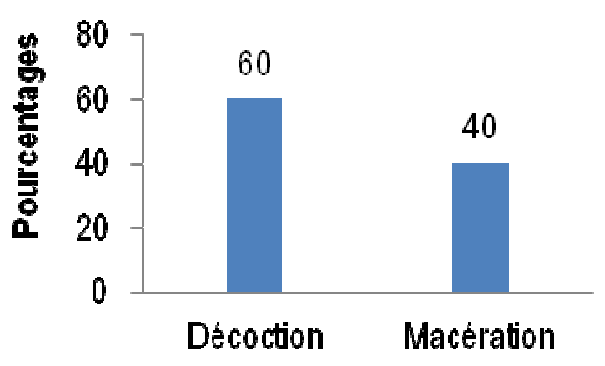

Mode de préparation

\section{Modes de préparation}

Figure 5 : Différents modes de préparation de Momordica charantia à Cotonou

- Voies d'administration: De l'analyse de cette figure, de toutes les méthodes d'utilisation, les voies d'administration dominantes sont les voies orale (50\%) et cutanée (40\%). Les méthodes d'ablution sont utilisées pour les rites surtout (10\%). Ceci est confirmé par les travaux de Dibong et al., (2011) qui affirme que la voie 
$\%$ dans l'utilisation des plantes médicinales.

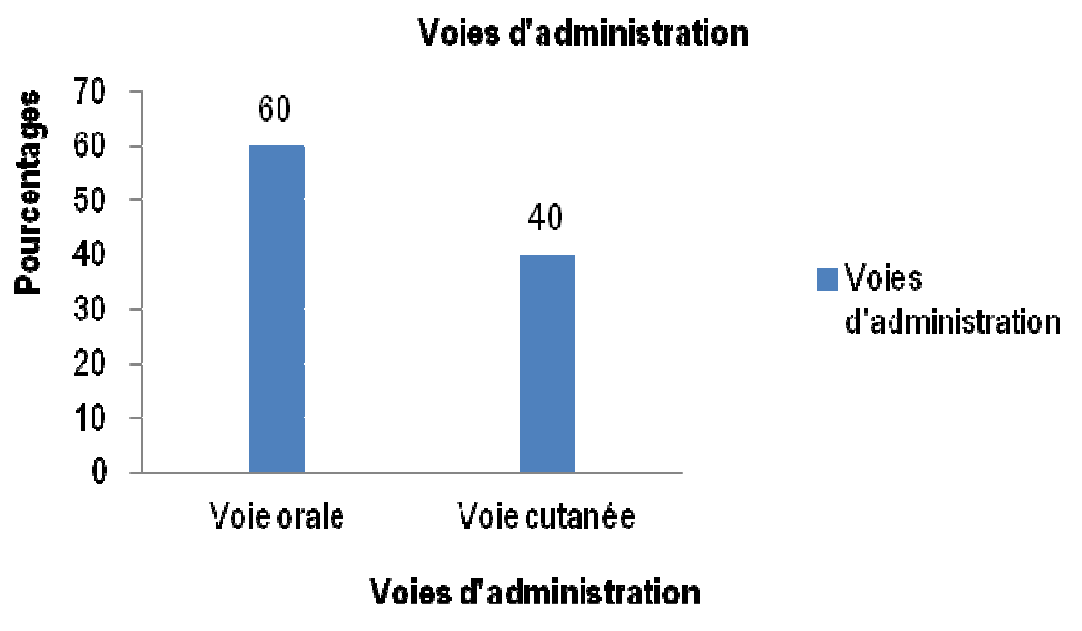

Figure 6 : Différentes voies d'administration de Momordica charantia à Cotonou

- $\quad$ Les types d'usages: La figure 6 traduit le pourcentage des différents types d'usages. L'observation de cette figure montre que l'usage thérapeutique prédomine avec $75 \%$ et $25 \%$ pour l'usage rituel. C'est donc une plante plus thérapeutique que rituelle. En dehors du Benin et du Togo où Momordica charantia est aussi utilisé à des fins rituelles, elle intervient surtout dans les thérapies de diverses maladies. L'usage thérapeutique au Benin prédomine avec un pourcentage de $75 \%$, suivi de l'usage rituel $25 \%$. Momordica charantia est une plante qui intervient dans le traitement de différentes maladies et pour ces diverses indications, différents organes sont utilisés, préparés de différentes manières, avec différentes voies d'administration. Momordica charantia est également utilisé dans les rites traditionnels.

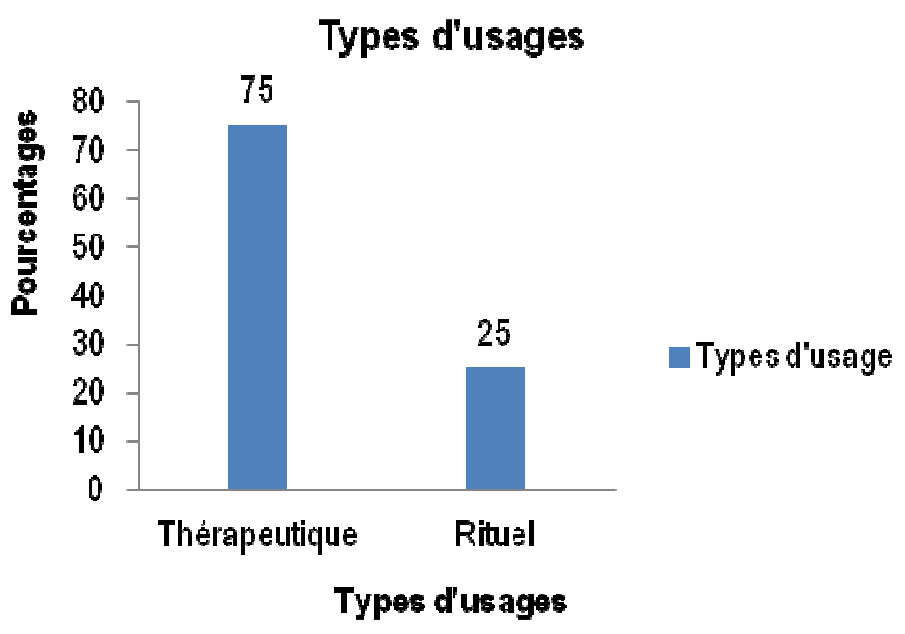

Figure 7 : Différents usages de Momordica charantia au Cotonou.

De l'étude phytochimique : Les résultats de l'analyse phytochimique sont présentés dans le tableau 1. Ce tableau montre les résultats des différents tests phytochimiques. II ressort de son analyse, que neuf groupes de composés chimiques sont caractérisés. Ces résultats sont conformes à ceux obtenus par Ezenwali, (2013) et Olalekan et al., (2013). La présence d'alcaloïdes, de flavonoïde et de tanins révélés dans ces différents extraits de la plante serait à l'origine des activités antimicrobiennes observées. Ces résultats 


\section{Johnson et al. J. Appl. Biosci. 2016 Étude ethnobotanique et phytochimique de Momordica charantia Linn (Cucurbitaceae) à Cotonou au Bénin}

permettent de valider l'usage de cette plante dans le

(Gbogbo et al, 2013).

traitement des maladies dues aux microorganismes

Tableau 1 : Résultat du dosage phytochimique de Momordica charantia

\begin{tabular}{|c|c|c|c|c|}
\hline Groupes chimiques & \multicolumn{3}{|c|}{ Différents composés recherchés } & Résultats \\
\hline \multirow{10}{*}{ Composés phénoliques } & \multirow[t]{2}{*}{ Tanins } & \multicolumn{2}{|c|}{ T- catéchiques } & ++ \\
\hline & & \multicolumn{2}{|c|}{ T- Galliques } & +++ \\
\hline & \multirow{2}{*}{ Flavonoïdes } & Flavones & & +++ \\
\hline & & Leucoantho & & ++ \\
\hline & \multicolumn{3}{|l|}{ Anthocyanes } & - \\
\hline & \multirow{4}{*}{$\begin{array}{l}\text { Dérives } \\
\text { anthracéniques }\end{array}$} & \multicolumn{2}{|l|}{ Libres } & ++ \\
\hline & & \multirow[t]{3}{*}{ Combinés } & Composés réducteurs & + \\
\hline & & & O-Hétérosides & - \\
\hline & & & C-Hétérosides & - \\
\hline & \multicolumn{3}{|l|}{ Coumarines } & - \\
\hline Composés azotés & \multicolumn{3}{|l|}{ Alcaloïdes } & + \\
\hline \multirow{4}{*}{ Stéroïdes et triterpènes } & \multicolumn{3}{|l|}{ Stéroïdes } & ++ \\
\hline & \multicolumn{3}{|l|}{ Triterpènes } & - \\
\hline & \multicolumn{3}{|l|}{ Saponosides } & - \\
\hline & \multicolumn{3}{|l|}{ Cardenolides } & - \\
\hline \multicolumn{4}{|l|}{ Mucilages } & +++ \\
\hline \multicolumn{4}{|l|}{ Dérivés cyanogéniques } & - \\
\hline
\end{tabular}

La présence, des stéroïdes, est conforme aux résultats de Duquesne (2006) qui affirme que le principal composé stéroïdien présent dans Momordica charantia est la Charantine. Notons la présence particulière des stéroïdes et des alcaloïdes dans Momordica charantia. Duquesne, (2006) a également obtenu les mêmes résultats, sauf que cette dernière a identifié en plus, les triterpènes. Cette différence pourrait être due à l'origine géographique et aux divergences génétiques des échantillons utilisés. Les mucilages, substances que contient Momordica charantia en proportion considérable,sont des fibres solubles possédant aussi plusieurs propriétés médicinales. Ils régularisent le taux de cholestérol, et luttent contre le cancer et le diabète (Dougnon, et al., 2012). Les échantillons de Momordica charantia dosés contiennent aussi des composés réducteurs. Ces derniers sont des monosaccharides et des disaccharides (Otshudi et al., 2000). Le rôle antipaludéen des flavonoïdes continue d'opposer certains auteurs : Morel (2011), s'oppose à Ueno et al., (1996), qui affirmait que Momordica charantia ne possède aucune activité de ce genre.

De l'étude des Éléments Traces Métalliques et quelques éléments nutritionnels : Étant donné que Momordica charantia, en plus d'être une plante médicamenteuse, est prisé parmi les produits alimentaires de certaines nations, nous utilisons les normes de l'OMS pour les aliments.

Tableau 2 : Résultat des dosages métaux lourds et de quelques éléments nutritionnels.

\begin{tabular}{l|l|l|l}
\hline Types de dosages & Éléments recherchés & Résultats (ppm) & Normes OMS (ppm) \\
\hline \multirow{3}{*}{ Éléments Traces } & Dithizone de Plomb & 2,7 & 0,3 \\
\cline { 2 - 4 } & Cadmium Dithizone & 1,26 & 0,2 \\
\hline \multirow{4}{*}{ Substances nutritives } & Fer & 0,28 & - \\
\cline { 2 - 4 } & Cuivre & 51,573 & - \\
\cline { 2 - 4 } & Chlorure & 155,7 & - \\
\cline { 2 - 4 } & Potassium & 0,6 & - \\
\cline { 2 - 4 } & Manganèse & 0,029 & - \\
\cline { 2 - 4 } & Protéine & 171 & - \\
\hline
\end{tabular}


Le tableau 2, révèle la présence en quantité importante de plomb et cadmium dans l'extrait de la poudre de Momordica charantia. Quantités bien au-delà des valeurs admises par l'OMS. Les concentrations en plomb et en cadmium des organes des plantes prélevés, sont respectivement 9 fois et 6,3 fois supérieures aux normes de l'OMS. Les concentrations du plomb dans Momordica charantia sont supérieures à celles obtenues par Dougnon, et al., (2013), dans Solanum macrocarpon ( 1,2 ppm pour les feuilles et 1,6 ppm pour les fruits), et proche de celles trouvées par Montcho, (2012),. dans les plantes médicinales antipaludiques. En ce qui concerne le cadmium, Momordica charantia contient une quantité bien au-delà des valeurs de Dougnon, et al., (2013), et de Montcho, et al., (2012). Ceci pourrait s'expliquer par l'environnement de plus en plus dégradé du fait de la mauvaise gestion des déchets dangereux, que ce soit en ville ou dans les campagnes. Les appareils électroménagers envahissent même nos campagnes et les déchets issus de leurs utilisations, polluent

\section{CONCLUSION}

La médication par les plantes est une thérapie ancestrale et millénaire. Ce travail sur Momordica Charantia prélevé à Cotonou, a permis de connaitre les vertus thérapeutiques et les considérations liées à cette espèce. La population cotonoise fait usage de cette espèce dans la prévention ou la guérison de plusieurs grands types de maladies ou d'affections. La diversification dans

Contribution des auteurs : Johnson R. C., Houéto E. E., Kpètèhoto H. W., Boni G. ont assuré la conception, la réalisation et la rédaction de l'étude. Dougnon $\mathrm{V}$. $T$., Pognon E., Assogba F. ont contribué à la Rédaction et

\section{BIBLIOGRAPHIE}

Agbankpé A. J. A., Dougnon T. V., Bankolé H. S., Yèhouénou B., Yèdomonhan H. (2014). Étude ethnobotanique des légumes feuilles thérapeutiques utilisés dans le traitement des diarrhées au sud-Bénin. International Journal of Biological and Chemical Sciences 8 (4): 17841795. 12 pages.

Akomagni L. A. (2006). Monographie de la commune de Cotonou. Afrique conseil. Mission de décentralisation. Programme d'Appui au Démarrage des Communes. 47 pages.

Bitsindou M. (1986). Enquête sur la phytothérapie traditionnelle à Kindamba et Odzala (Congo) et analyse de convergence d'usage des plantes dangereusement les sols. Le trafic de l'essence frelatée, le trafic routier, les déchets municipaux, industriels, agricoles et artisanaux sont les sources de contamination les plus importantes (Soclo et al., 2000 ;. Youssao, 2011). Le tableau 2, montre également la présence en fraction importante de quelques éléments nutritionnels dans les extraits de la plante, avec une remarquable présence des protéines et des chlorures. Les résultats ont révélé que $100 \mathrm{~g}$ de la plante entière renferme $171 \mathrm{mg}$ de protéine, $0,6 \mathrm{mg}$ de potassium et $0,07 \mathrm{mg}$ de zinc. L'étude comparative de ces valeurs à celles obtenues par Duquesne (2006), sur la même plante, montre que, Momordica charantia vendu sur les marchés de Cotonou renferme plus de protéine que les fruits et les feuilles, objets de ses études. Le potassium, le zinc et le fer sont également présents, mais à des quantités faibles par rapport aux espèces étudiées par Duquesne, (2006). D'autres substances telles que le manganèse et les chlorures sont quantifiées. Cela indique Momordica charantia comme un potentiel complément alimentaire.

l'utilisation des organes de cette plantes est liée à celle de ces organes végétaux. L'élargissement des connaissances sur les vertus de cette plante serait un facteur de motivation pour sa domestication à travers sa culture. Ces résultats permettent de justifier l'utilisation de cette plante dans la pharmacopée traditionnelle.

relecture de l'étude. Loko F., Boko M. et Gbénou J. ont supervisé les travaux.

Remerciement : Nous remercions sincèrement tous ceux qui ont participé à la réalisation de ce travail.

médicinale en Afrique centrale. Mem. Doc (inéd.). Univ. Libre de Bruxelles. 482 pages.

Dibong.S. D., Mpondo M. E., Ngoye A. K., Betti J. L., (2011). Ethnobotanique et phytomédecine des plantes médicinales de Douala. 2496-2507...11 pages.

Dougnon T. V., (2012). Évaluation des propriétés hémostatiques de la sève de Jatropha multifida L. (Euphorbiaceae). Mémoire pour l'obtention du diplôme de master professionnel option. Génie de Biologie Humaine (GBH), spécialitéTechnologie Biomédicale(TB). EPAC / UAC. 75 pages.

Dougnon V., (2013). Utilisation des fientes de poulets, des eaux de marécage en agriculture urbaine et 
qualité sanitaire de Solanum macrocarpon $L$. (Solanaceae). Diplôme d'Études Approfondies (D.E.A) en Environnement, Santé et Développement Durable. 71 pages.

Duquesne E. H. (2006). Melon amer Momordica charantia. 2014/mai/memoire_melon-amere.pdf.

Ezenwali M. O. (2013). Phytochemical analysis and the anti-inflammatory activities of methanol extract of Crateva adansonii. By uwah lynda ogechi.BC/2009/262. Faculty of Natural Science Caritas University, Amorji-Nike. Emene, Enugu State.

INSAE, (2012), 4 ème Recensement.général de la population RGPH4 ; Résultat provisoire.

Kougnimon F., Dougnon V., Anago E., Bankolé H., Soumanou M., Loko F. (2015). Propriétés biologiques et pharmacologiques de Terminalia superba Engl. et Diels (Combretaceae): Synthèse bibliographique. 164 - 176. 13 pages.

Gbogbo K. A., Amègninou A., Woegan Y. A., Amana K. E.,.Hoekou P. Y., Batawila K., Koumaglo K., Akpagana K. (2013). Évaluation de l'activité antimicrobienne de Momordica charantia (Cucurbitaceae), Psidium guajava (Myrtaceae) et Pteleopsis suberosa (Combretaceae). European Scientific Journal Décembre 2013 édition vol.9, No.36 ISSN : 1857 - 7881.

Kpodékon M. T., Boko K. C., Mainil J. G., Farougou S., Sèssou P.,.Yèhouenou B., Gbénou J., et al. (2014). Composition chimique et test d'efficacité in vitro des huiles essentielles extraites de feuilles fraîches du basilic commun (Ocimum basilicum L.) et du basilic tropical (Ocimum gratissimum L.) sur Salmonella enterica sérotype Oakland et Salmonella enterica sérotype Legon. 25 pages.

Lahsissène H., Kahouadji A., Tijane M., Hseini S. (2010). Catalogue des plantes médicinales utilisées dans la région de Zaër (Maroc Occidental) Lejeunia, 186, 1 - 27.

Lhuillier M. A. (2007). Contribution à l'étude phytochimique de quatre plantes malgaches: Agauria salicifolia Hook.F ex Oliver, Agauria polyphylla Baker (Ericaceae), Tambourissa trichophylla Baker (Monimiaceae) et Embelia concinna baker (Myrsinaceae). Thèse de doctorat de l'Institut National Polytechnique de Toulouse. Spécialité, Sciences des Agro ressources. 214 pages.

Montcho S. (2012). Les plantes médicinales antipaludiques : une autre voie d'exposition aux métaux toxiques à Cotonou (Bénin) ? Mémoire de Master $\left(\mathrm{M}_{\mathrm{sc}}\right)$ Environnement, Santé et Développement Durable. CIFRED / UAC. 62 pages.

Morel S. (2011). Étude phytochimique et évaluation biologique de Derris ferruginea Benth. (Fabaceae). Thèse de doctorat, Université d'Angers. 245 pages.

Olalekan A. J., Adékunlé A.I., Labunmi L. (2013). Chemical and phytochemical composition of wild lettuce (Launaea taraxacifolia). Journal of Applied Phytotechnology in Environmental Sanitation, 2 (1):25-30. 06 pages.

Okafor J. C., Ham R. (1999). Identification, utilisation et conservation des plantes médicinales dans le sud-est du Nigeria. Thèmes de la biodiversité africaine, $\mathrm{N}^{\circ} 3$. (anglais / français). 08 pages.

OMS (2013). Renforcement du rôle de la médecine traditionnelle dans les systèmes de santé : une stratégie pour la région Africaine. Rapport du Secrétariat.

Otshudi A. L., Foriers A., Vercruysse A., Van Zeebroeck A., Lauwers S. (2000). In vitro antimicrobial activity of six medicinal plants traditionally used for the treatment of dysentery and diarrhoea in Democratic Republic of Congo (DRC). Pub Med, Apr 7(2):167 - 72.

Salhi S. (2010). Études floristique et ethnobotanique des plantes médicinales de la ville de Kénitra (Maroc).

Soclo H. H., Guarigues P., Ewald M. (2000). Origin of Polycyclic Aromatic Hydrocarbons (PAHs) in Coastal Marine.

Soh N. P., Benoit V. (2007). Are West African plants a source of future antimalarial drugs? Journal of Ethnopharmacol ; 114:130 - 40.

Tahri N.,.EL Bastia A., Zidane L., Rochidi A., Douira A..(2012). Étude ethnobotanique des plantes médicinales dans la province de Settat (Maroc). Faculty Kastamonu Univ., Journal of Forestry. 12 (2): 192-208. 16 pages.

Ueno H. M., Doyama J. T., Padovani C. R., Salata E. (1996). Effect of Momordica charantia L. in mice infected with Plasmodium berghei.

Youssao A., Soclo H. H., Bonou C., Fayomi B. (2011). Évaluation de la bioaccumulation du plomb dans les espèces animales marines et identification des sources de contamination métallique par.une analyse multi élémentaire en métaux $(\mathrm{Al}, \mathrm{Cd}, \mathrm{Cr}, \mathrm{Cu}, \mathrm{Pb})$ dans les eaux côtières $\mathrm{du}$ Bénin. Journal Home Vol 5, No 1. 